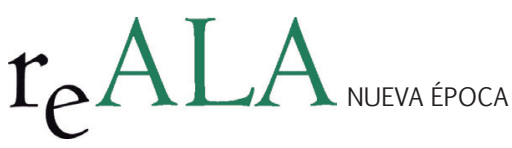

REALA, n 1, enero-junio 2014, ISSN: 1989-8975

DOI: http://dx.doi.org/10.24965/reala.voi1.10144

\title{
Estrategia de desarrollo de la Zona Norte del Estado de Jalisco desde la perspectiva institucional
}

\author{
Mónica Isabel García Mora \\ Centro Universitario de Ciencias Económico Administrativas, Universidad de Guadalajara \\ monica.garcia.m@hotmail.com \\ José G.Vargas-Hernández \\ Centro Universitario de Ciencias Económico Administrativas, Universidad de Guadalajara
} josevargas@cucea.udg.mx

Recibido: 12 de diciembre de 2013 Aceptado: 10 de diciembre de 2013

Resumen

El proceso de regionalización en Jalisco ocurrido en la década de los noventa, dio lugar a la descentralización de las regiones y a la formulación de un plan regional de desarrollo para cada una de ellas. Actualmente y a pesar de las medidas tomadas al establecer los planes regionales, se observa que existen disparidades en el desarrollo de las regiones, siendo la más afectada, la Zona Norte del Estado. El objetivo de este trabajo, es analizar como factor clave, la relación de las instituciones con el desarrollo de la Zona Norte del Estado de Jalisco, haciendo un breve análisis del papel de las instituciones desde la perspectiva económica, política y social. El método empleado será la revisión de la literatura existente sobre la teoría institucional y el desarrollo de la Zona Norte del Estado.Se concluye señalando como estrategia para impulsar el desarrollo, la evaluación y el fortalecimiento de las instituciones que intervienen en este proceso.

Palabras clave

Desarrollo, desarrollo regional, instituciones, región, zona norte del Estado de Jalisco

\section{Strategy of Development of the Zone North of the State of Jalisco from the Institutional Perspective}

\section{Abstract}

The regionalization process in Jalisco occurred in the nineties leading to the decentralization of regions and formulating a regional development plan for each of them. Currently, despite the measures taken to establish regional plans, it appears that there are disparities in regional development, being the most affected, the North Zone of the State. The aim of this paper is to analyze a key factor, the ratio of the institutions with the development of the Northern region of the State of Jalisco, with a brief analysis of the role of institutions from economic, political and social perspectives. The method used is a review of the existing literature on institutional theory and the development of the Northern Zone of State. It concludes by stating as a strategy to promote the development, evaluation and strengthening of the institutions involved in this process.

Keywords

Development, Regional Development Institutions, Region, Northern State of Jalisco. 


\section{INTRODUCCIÓN}

El desarrollo de las regiones se ha vuelto un tema muy importante en los últimos años, y es que hablar de desarrollo, implica pensar en mejoras en la calidad de vida y mayor bienestar social de un país o región. Formalmente, el desarrollo regional se define como "un proceso de crecimiento y cambio estructural que, mediante la utilización del potencial de desarrollo existente en el territorio, conduce a elevar el bienestar de la población de una localidad o región" (Diez, 2004).

Es bien sabido que por cuestiones históricas, el desarrollo en México no se ha dado conforme a las expectativas y las necesidades del país, aunado a esto, existen disparidades en el desarrollo entre las regiones. Por ejemplo, en datos revelados por el Instituto Nacional de Estadística y Geografía (INEGI, 2010), Jalisco es el cuarto estado con más participación en el Producto Interno Bruto (PIB), es decir, el cuarto estado más productivo de la República Mexicana, sólo después del D.F, Estado de México y Nuevo León, sin embargo, aunque este indicador económico es bueno, en contraste tenemos el Índice de Desarrollo Humano (IDH, 2009), el cual coloca a Jalisco en el lugar 14 en relación al índice de ingreso comparado con los demás estados de la República.

El IDH Jalisco evalúa también las 12 regiones que componen Estado, es aquí donde observamos que el desarrollo se da de una forma muy heterogénea en Jalisco y donde se pueden observar las disparidades en el desarroIlo, especialmente en la Zona Norte de Jalisco, la cual en la evaluación del IDH Jalisco coloca a esta región en el último lugar. Por esta razón, nace el interés de analizar en este trabajo las disparidades en el desarrollo de la Zona Norte de Jalisco, vistas desde la influencia de las instituciones en el desarrollo de la región.

\section{LA REGIONALIZACIÓN EN JALISCO}

A mediados de la década de los 9o, los cambios políticos en el estado de Jalisco trajeron una nueva forma de organizar los planes de desarrollo económico, que se inician con la segmentación de zonas geográficas cercanas y con características homogéneas denominadas regiones.

En 1997, se inició un proceso de regionalización en el Estado de Jalisco, este proceso de regionalización fue consecuencia de la apertura del país con miras a aprovechar las ventajas del proceso de globalización y hacer frente a las desigualdades regionales. La Estrategia de regionalización en Jalisco, dio origen a la concentración de los 124 Municipios que conforman el Estado en doce regiones administrativas con el objetivo de "fomentar el proceso de descentralización y desconcentración hacia las regiones, e Impulsar el proceso de planeación social, integral, participativa y estratégica para desarrollar las regiones", Plan Estatal de Desarrollo (PED, 2030, 16-17).

Sin embargo, a pesar de que desde 1995 se han implementado Planes Estatales de Desarrollo en Jalisco para mitigar las disparidades de desarrollo entre las regiones, aun quedan regiones con visibles muestras de rezago, "mientras la desigualdad nacional pudo haberse reducido hasta un 15\% entre 2000 y 2005, en Jalisco aumentó $62 \%$ en el mismo periodo" (IDH, 2009). Un claro ejemplo, al hablar de las disparidades regionales, es el caso de la Región Norte del Estado de Jalisco.

\section{GENERALIDADES DE LA REGIÓN NORTE DEL ESTADO DE JALISCO}

La Región Norte del Estado de Jalisco, se ubica en la punta Norte del Estado de Jalisco (ZNEJ), su territorio equivale a $10,305.46 \mathrm{~km} 2$, que corresponde al $12.86 \%$ de la superficie total del Estado de Jalisco. Limita al norte con los estados de Nayarit y Zacatecas; al este con el Estado de Zacatecas; al oeste el Estado de Nayarit y al sur, el Estado de Zacatecas y los Municipios Jaliscienses de Tequila y Hostotipaquillo. La región está integrada por 10 Municipios: Bolaños, Chimaltitán, Colotlán, Huejúcar, Huejuquilla el alto, Mezquitic, San Martín de Bolaños, Santa María de los Ángeles, Totatiche y Villa Guerrero.

La Zona Norte del Estado de Jalisco, "es una de las regiones de Jalisco con más potencialidad y, sin embargo, de las más rezagadas" (Romero, 2009). Como un panorama muy general, podemos mencionar que la población de la región representa tan sólo el 1.04 \% de la población estatal. El 60\% de los municipios están catalogados como Rurales y el $40 \%$ como Semiurbano. La región norte del estado de Jalisco cuenta con una fuerte presencia de culturas indígenas, según INEGI (2010) 4 de los 10 municipios de la ZNEJ, tienen el mayor porcentaje de la población de habla indígena del Estado, Mezquitic con $75.9 \%$ y Bolaños con $64.4 \%$; les siguen los municipios de Huejuquilla el Alto con 6.5\% y Villa Guerrero con 5.5\%, prevaleciendo la cultura del pueblo Huichol en Mezquitic y Bolaños principalmente.

En cuanto a índices de marginación, la ZNEJ es la zona más marginada de la entidad, ya que tiene un muy alto grado de marginación, este índice de marginación nos permite diferenciar las carencias que padece la población, considera la falta de acceso a la educación, la residencia en viviendas inadecuadas, la percepción de ingresos monetarios insuficientes y la residencia en localidades pequeñas (CONAPO, 2010). A pesar de estas cifras no muy 
alentadoras, la Zona Norte de Jalisco cuenta con un gran potencial de recursos, principalmente recursos naturales, que pueden ser considerados como estrategia para impulsar el desarrollo endógeno de la Zona.

Esta Zona posee recursos hidrológicos, proporcionados por los ríos Bolaños, que pasa de norte a sur y al poniente de la cabecera, el río Camotlán, que con la misma dirección toca el poniente de la superficie municipal, hay también pequeños manantiales. Entre los principales cerros y serranías están, La Palma, Violeta, El Caimán, El Aguacate, Pitacho de patoles, Los Sabinos, El Eslabón, Tapaiste, La Campana y Guajolotes.

Entre los principales atractivos turísticos y culturales de la Zona Norte de Jalisco, se encuentran los que se recogen en la tabla 1.

TABLA 1

ATRACTIVOS TURÍSTICOS Y CULTURALES DE LA ZONA NORTE DE JALISCO

\begin{tabular}{|c|c|c|}
\hline Municipio & Atractivo Turístico & Descripción \\
\hline Bolaños & La Casa de la Condesa & $\begin{array}{l}\text { Mansión barroca de fines del siglo XVIII, ubicada en la ca- } \\
\text { Ile } 16 \text {, \# } 39 . \text { Fachada de dos niveles con esgrafiados con es- } \\
\text { cenas de la vida diaria y figuras fitomorfas en sus cenefas, } \\
\text { así como decoración esgrafiada en sus enjarres originales. }\end{array}$ \\
\hline \multirow[t]{2}{*}{ Chimaltitán } & $\begin{array}{l}\text { Zona Arqueológica "Los Cerri- } \\
\text { tos" }\end{array}$ & $\begin{array}{l}\text { En este sitio se encuentran piedras de cantera grabadas } \\
\text { en relieve con algunos signos y dibujos. }\end{array}$ \\
\hline & Piedras Largas & $\begin{array}{l}\text { Paraje montañoso de pino y roble con figuras caprichosas } \\
\text { formadas por la naturaleza en diferentes tamaños, en un } \\
\text { área aproximada de } 2 \text { hectáreas }\end{array}$ \\
\hline Colotlán & Cueva de la Novia & $\begin{array}{l}\text { Es una cueva que mide } 8 \text { metros de alto por } 12 \text { de ancho, } \\
\text { desde este sitio se puede admirar todo el municipio de } \\
\text { Colotlán, Santa María de los Ángeles, Huejúcar, Momax y } \\
\text { Tlaltenango, además: la carretera, Colotlán, San Nicolás, } \\
\text { El Carrizal y la presa de Boquilla de los Pérez. }\end{array}$ \\
\hline Huejuquilla el alto & $\begin{array}{l}\text { Zona Arqueológica "Cerrito } \\
\text { de Hiztle" }\end{array}$ & $\begin{array}{l}\text { En el lugar se encuentran restos de culturas precolombi- } \\
\text { nas. }\end{array}$ \\
\hline Mezquitic & Mirador Mezquitic & $\begin{array}{l}\text { Se localiza aproximadamente a } 5 \text { kilómetros de distancia } \\
\text { de la cabecera municipal de Mezquitic, sitio destinado } \\
\text { como mirador, se domina un gran cañón y parte del valle } \\
\text { de Mezquitic, de igual manera se aprecia también el río } \\
\text { Bolaños. Ideal para desarrollar el paisajismo y la medita- } \\
\text { ción. }\end{array}$ \\
\hline $\begin{array}{l}\text { San Martín de Bo- } \\
\text { laños }\end{array}$ & Planta Minera “El Pilón” & $\begin{array}{l}\text { Es una planta procesadora de plata, la cual se puede cono- } \\
\text { cer a través de visitas guiadas donde se muestra el proce- } \\
\text { so a que es sometido este bello metal, con un horario de } \\
\text { 8:00 a 17:00 horas de martes a viernes. }\end{array}$ \\
\hline $\begin{array}{l}\text { Santa María de los } \\
\text { Ángeles }\end{array}$ & Presa “Boquilla de Zaragoza” & $\begin{array}{l}\text { Se localiza al norte de la cabecera, se puede practicar la } \\
\text { pesca de lobina, carpa y bagre durante todo el año. En } \\
\text { este sitio es posible observar pato bobo, papello. Tam- } \\
\text { bién el paisaje de lomas suaves cubiertas por bosque } \\
\text { tropical. Cuenta con algunos espacios para acampar en la } \\
\text { parte norte y oeste del embalse. }\end{array}$ \\
\hline Totatiche & La Cueva de las Patas & $\begin{array}{l}\text { Es un misterioso y antiquísimo lugar enclavado en la ba- } \\
\text { rranca de la comunidad de El Canjilon, petroglifos que aún } \\
\text { conservan y que datan de } 8,000 \text { años de antigüedad. }\end{array}$ \\
\hline Villa Guerrero & Cascadas “La Pila del Diablo” & $\begin{array}{l}\text { Se encuentra en el poblado de Patahua a } 8 \text { kilómetros } \\
\text { por terracería. Son albercas naturales formadas por los } \\
\text { peñascos que lo circunden su vegetación de sabino, y en } \\
\text { tiempo de lluvias se forman pequeñas cascadas. }\end{array}$ \\
\hline
\end{tabular}

FUENTE: Elaboración propia con datos de SEIJAL (2012). 
Estos son sólo algunos de los muchos atractivos turísticos, arqueológicos y culturales que podemos encontrar, esta zona es rica en recursos naturales, privilegiada por sus paisajes, es una zona ideal para fomentar el ecoturismo.

\section{IMPORTANCIA DE LAS INSTITUCIONES EN EL DESARROLLO}

El gran problema que acosa a la actual literatura ortodoxa sobre las instituciones y el desarrollo es su incapacidad para distinguir claramente entre las formas y las funciones de las instituciones (Chang, 2006). Sin embargo, para este trabajo, tomaremos la definición de North (1990) quien define las instituciones como las restricciones humanamente planeadas que estructuran la interacción de las personas, y son conocidas informalmente como "reglas del juego". Las instituciones son clave para asegurar que los esfuerzos de desarrollo sean sostenibles. Ya que son las instituciones las que regulan y administran la forma de llevar a cabo las acciones que contribuyen con el desarrollo de una región.

Acemoglu (2003, p. 27) encontró lo siguiente:

"Un factor primordial que explica las causas fundamentales de las diferencias en la prosperidad de los países son las instituciones. Las tres características clave de las instituciones buenas son la aplicación de los derechos de propiedad a un segmento amplio de la sociedad, de tal manera que varias personas tengan incentivos para invertir y participar en la actividad económica; límites a las acciones de las elites, los políticos y otros grupos poderosos, para evitar que expropien los ingresos y las inversiones de otros o que creen condiciones que les favorezcan; y cierto grado de igualdad de oportunidades para segmentos amplios de la sociedad, a fin de que las personas puedan invertir, especialmente en capital humano, y dedicarse a actividades económicas productivas".

Al respecto de los derechos de propiedad, North y Thomas (1973, p. 8) señalan que la creación, especificación y aplicación de los derechos de propiedad son costosas, y que estos costos dependen del estado de la tecnología y de la organización; los gobiernos asumen la protección y aplicación de los derechos de propiedad porque ellos pueden hacerlo a costos menores que los grupos privados que podrían organizarse voluntariamente.

Como señaló Bandeira (2009), luego de la aparición del famoso libro de North sobre las instituciones y el desarrollo económico en 1990, del fracaso de las políticas de ajuste estructural de los ochenta y los noventa, y de la contrastación de las tesis de North con la evidencia empírica, el nuevo paradigma es que las instituciones son la clave del desarrollo económico de las naciones.

Aunque el Estado es quien está al frente de las instituciones es importante que los actores que lo integran no abusen del poder que tienen, en este sentido, las instituciones involucradas en el desarrollo, principalmente el gobierno, debe ser capaces de establecer y aplicar las acciones encaminadas al desarrollo con el único fin de propiciar el bienestar social, sin desviarse de éste.

La igualdad de oportunidades entre los miembros de la sociedad, generará una actitud proactiva hacia el desarrollo de su comunidad, este apoyo es importante como base para establecer políticas de desarrollo en las que es indispensable la participación de los miembros de la sociedad.

Por último, debo destacar que que la relevancia estratégica de las instituciones en los procesos de desarrollo reside en que permite reducir los costes de transacción y producción, aumenta la confianza entre los actores económicos, estimula la capacidad empresarial, propicia el fortalecimiento de las redes y la cooperación entre los actores y estimula los mecanismos de aprendizaje y de interacción (Rodríguez Pose, 1998).

\section{LA RELACIÓN DE LAS INSTITUCIONES CON EL DESARROLLO DE LA ZONA NORTE DE JALISCO}

Desde el marco teórico antes planteado, me parece conveniente hacer un breve análisis de las instituciones de la Región Norte de Jalisco. Para efectuar dicho análisis, clasificaremos las instituciones desde el punto de vista económico, político, y social.

\section{INSTITUCIONES ECONÓMICAS}

De acuerdo con José Antonio Ramírez (citado en Spiller, 2010) En el Norte de Jalisco, “hay una percepción de las instituciones, muy negativa. Los programas para mejorar el nivel económico, no son apoyados por la población, ni son impulsados por el gobierno".

Un crecimiento económico dinámico debe ir acompañado de márgenes satisfactorios de equidad, igualdad de oportunidades y protección social, para esto es importante la participación ciudadana en las decisiones median- 
te consultas públicas por parte de las instituciones. No sólo se debe elaborar un Plan de desarrollo basándose en cifras y números sino también debe incluirse las necesidades evaluadas desde el punto de vista de la sociedad para involucrarlos en el proceso y obtener mejores resultados.

Por mencionar un caso, uno de los principales problemas que tiene la Zona Norte (80\% de los municipios) es la tala inmoderada y en segundo lugar pérdidas de la vegetación por incendios forestales. Sabiendo ya que los recursos naturales de la Zona son recursos potenciales para propiciar el desarrollo, la pérdida de estos constituye un gran desafío para las autoridades a las que les falta regular de manera más fuerte esta situación; las cifras no mienten, los municipios de Colotlán, Bolaños, Huejúcar, Huejuquilla, Mezquitic, San Martín de Bolaños, Totatiche y Villa Guerrero principalmente son los más afectados (PED, 2030, p.95).

Es importante que las autoridades competentes, consideren el aprovechamiento de los recursos naturales, ya que éstos pueden dar pie a un desarrollo endógeno. Esto es, "si la comunidad local es capaz de liderar el proceso de cambio estructural, nos encontramos ante un proceso desarrollo local endógeno” (Vázquez Barquero, 2000).

Una propuesta para promover el crecimiento económico desde la perspectiva de desarrollo endógeno, sería promover el Ecoturismo, como estrategia de promoción de los recursos turísticos natural y cultural de la región. Sin embargo, si las autoridades no son fuertes al procurar la preservación de los recursos naturales, seguirá deforestándose la vegetación y esta opción turística no sería viable.

Además de los recursos naturales, otra alternativa es cuidar los atractivos culturales como son las actividades realizadas por los huicholes, principalmente las artesanías elaboradas a mano, las cuales son distintivas de su cultura y sus costumbres.

Las autoridades deben apoyar a la cultura Huichol a aprovechar la elaboración y la comercialización de artesanías desde la perspectiva del marco (VRIO) la cual es una visión basada en los recursos que se concentra en los aspectos de valor, rareza, la imitación y la organización de los recursos y capacidades (Peng 2010). En este sentido, las artesanías tienen una ventaja competitiva, ya que son productos hechos a mano y raros por el hecho de que ya cada vez es menos cotidiana en las ciudades la presencia de culturas indígenas. Aquí deberán actuar las autoridades para procurar que estos productos tengan un pago digno ya que es una actividad que crea empleos y es el sustento de muchas familias Huicholes.

\section{INSTITUCIONES POLÍTICAS}

Desde hace años la rama de la teoría económica que estudia los sistemas políticos -la teoría de la elección social- argumenta que en los sistemas democráticos los políticos tienden a escoger aquellas políticas que les reporten mayores beneficios electorales y aquellas que se ven presionados a tomar por grupos de interés con poder político (Bandeira, 2009).

La literatura señala que podría haber dos razones que explican la ineficiencia en el cumplimiento de los objetivos del desarrollo. El primero es que la realización de elecciones cada pocos años lleva a que los políticos den prioridad a los resultados de corto plazo, en perjuicio de las políticas y reformas institucionales necesarias para el desarrollo económico, que sólo dan resultados en el largo plazo. El segundo es que los grupos de interés tienen objetivos de más largo plazo, pues buscan garantizar sus ingresos futuros, pero buscan objetivos muy específicos, es decir, que no benefician al conjunto de la sociedad, para reducir los costos y aumentar los beneficios de su acción política (Olson, 1965).

La gobernabilidad de una región depende de los actores que se involucran en dicho proceso, los partidos políticos, el gobierno (de los tres niveles, municipal, estatal y federal), por ello es importante evaluar constantemente el papel que han jugado estas instituciones en el desarrollo basándose en la capacidad del sistema político para negociar coaliciones estables, políticas fuertes o la solidez de las instituciones públicas, la habilidad del gobierno y de los distintos sectores sociales para combinar adecuadamente las políticas de desarrollo.

Según Jacoby (citado en Chang, 2006) subraya el papel de la legitimidad en el proceso de cambio institucional. Una nueva institución no puede funcionar a menos que consiga cierto grado de legitimidad política entre los miembros de la sociedad. De aquí surge la importancia de crear un vínculo estado-sociedad para que se trabaje de manera conjunta y procurar el desarrollo en la Zona Norte del estado. Al respecto, hay que reconocer que se han hecho y tomado algunas acciones para lograr integrar a los habitantes de la ZNEJ al proceso político.

Por ejemplo, en estas próximas elecciones se trata incentivar a la población indígena que vive en la Zona Norte a integrarse al proceso político. En un artículo del Diario el Informador (Autor anónimo, 2012) dio a conocer que este 2012 es año electoral y la Zona Norte de Jalisco también va a votar ya que los pobladores no votarán de la manera tradicional, sino con una urna electrónica; o al menos es lo que pretende hacer el Instituto Electoral y de 
Participación Ciudadana (IEPC). Una comunidad beneficiada será la de San Andrés Cohamiata, o Tateikie, en lengua wixárica, que está en el Norte del Estado y pertenece al municipio de Mezquitic., para acceder a la comunidad se invierten alrededor de 10 horas, saliendo de Guadalajara, sin embargo, el IEPC capacitará a habitantes de esta comunidad y algunas otras de la Zona Norte para que conozcan el uso de los aparatos electrónicos que pretenden utilizar el 1 de julio.

Este es un esfuerzo importante del IEPC que ayudará a que la población de la zona norte, la cual tiene un alto porcentaje de población indígena a que pueda elegir a sus gobernantes y al sentirse tomados en cuenta, también se involucren activamente en el proceso de desarrollo.

Por último, cabe señalar que en la Zona Norte de Jalisco, 9 de los 10 municipios que conforman esta zona, están gobernados por el PAN, esto supondría una homogeneidad en la ideología del pensamiento político, lo cual debería ser una razón a favor para propiciar el desarrollo dentro de un nuevo marco administrativo eficiente. Sería importante que las instituciones políticas de la Zona Norte, aprovecharan que comparten ideología política e hicieran relaciones intergubernamentales entre los municipios que conforman la región para procurar el desarrollo conjunto de la zona.

\section{INSTITUCIONES SOCIALES}

Las instituciones y la sociedad se comportan de forma dinámica, pero a la par deben trabajar de forma armonizada para lograr los objetivos del desarrollo. Como ya se mencionó, es importante que la sociedad se involucre de forma activa en las cuestiones del desarrollo, una importante forma de participación es la evaluación de los programas y políticas públicas que se implementan en la Zona Norte con el objetivo de impulsar el desarrollo de la región.

Sin embargo, en la Región Norte existen bajos niveles de educación entre la población de 15 años, se sabe que la población de la Región Norte, es la que cuenta menor competitividad por no haber concluido la primaria el $28 \%$ de la población de 15 y más años de edad, el doble de la proporción en el estado (que es de 14.7\%), Aún más, cuatro de los municipios están por arriba de la proporción de la región: Santa María de los Ángeles, con 39.9\%; Chimaltitán, 35.1\%; Totatiche, 32.5\% y Huejúcar con 31.3\%. (PED, 2030, p.53). Estos datos nos dan un ligero panorama de la situación en la Zona Norte, es alto el porcentaje de la población que no concluyó ni siquiera la primaria por lo cual es difícil que esta proporción de la población con tan bajo nivel educativo se pueda involucrar de manera activa en las cuestiones del desarrollo en sus comunidades.

Sin embargo, el desarrollo de la Zona Norte ya no es de interés aislado, existen ya organizaciones y académicos que están constantemente preocupados por el desarrollo de esta región, de hecho ya existe un movimiento social que procura el desarrollo de esta zona: El 9 de junio fue creado el Movimiento Ciudadano del Norte de Jalisco, con la firma de un documento constitutivo que suscribieron 54 personas de diferentes ámbitos sociales, económicos, culturales y políticos creado ante las dificultades y sobre todo la inquietud de realizar más por la zona, y preocupado por que los candidatos a diputados locales y federales y a presidente municipal generen un compromiso con la región (Romero, 2009).

La creación de este movimiento es de vital importancia para el desarrollo de la región ya que las ONGs pueden acceder al desarrollo de programas estatales y, en algunas ocasiones, interactuar con el ámbito público en la elaboración e implementación de la política (Arroyo, 2010).

\section{CONCLUSIÓN}

Es importante que en la Zona Norte del Estado de Jalisco, se de un cambio institucional, es decir, una interacción entre instituciones y organizaciones para lograr los retos de desarrollo en la Región. Las instituciones entendidas como las reglas del juego, son un factor esencial en el proceso de desarrollo, sin embargo, deben complementarse con las organizaciones, estás organizaciones son los partidos políticos, las empresas, las familias, las Universidades, Organizaciones No Gubernamentales (ONG), Organizaciones Civiles, entre otros actores que pueden contribuir con el desarrollo de la región.

Las instituciones no se crean con el fin exclusivo de promover el desarrollo de la sociedad, también sirven al propósito de distribuir o consolidar el poder político y económico. Sin embargo, la clave es que las instituciones y las políticas económicas promuevan el bienestar de la ciudadanía y no la satisfacción de intereses políticos y económicos particulares (Bandeira, 2009).

Es importante que se fortalezcan las instituciones y se procure como objetivo principal los intereses y el bienestar social. Para esto se debe evaluar la eficacia de las instituciones y de los programas y políticas de desarrollo propuestas por el gobierno. 
La sociedad debe ser parte de este proceso al participar activamente en la evaluación y propuesta de los planes de desarrollo en la Zona Norte del Estado, ya que es la misma sociedad la que conoce las necesidades primordiales de su propia región.

Las organizaciones políticas tales como los partidos políticos y gobernantes de la región deben procurar el desarrollo viendo por los intereses de la región y no sólo emitiendo propuestas que en el corto plazo les garantizarán votos y la obtención del poder.

\section{BIBLIOGRAFÍA}

ACEMOGLU, D. (junio de 2003). "Raíz histórica: un enfoque histórico de la función de las instituciones en el desarrollo económico". Finanzas y desarrollo: publicación trimestral del Fondo Monetario Internacional y del Banco Mundial,40(2). Recuperado de: http://www.imf.org/external/pubs/ft/fandd/spa/2003/06/pdf/acemoglu.pdf

ARROYO, D. (2010). La Participación de la Sociedad Civil en el nivel local: experiencias regionales en Argentina. Gestión Cultural Lujan. Recuperado de: http://es.scribd.com/doc/26713466/Participacion-de-La-Sociedad-Civil-enDesarrollo-Local

ANÓNIMO (18 de mayo_2012)._Llevan la urna electrónica a la zona wixárica”. Diario el informador. Recuperado de: http://www.informador.com.mx/jalisco/2012/377020/6/llevan-la-urna-electronica-a-la-zona-wixarica.htm_

BANDEIRA, P. (2009). Instituciones y Desarrollo Económico. Un Marco conceptual. Revista de Economía Institucional, 11 (20). Recuperado de: http://www.economiainstitucional.com/pdf/No20/pbandeira20.pdf

CHANG, HA (2006). La relación entre las Instituciones y el desarrollo económico. Problemas Teóricos claves. Revista de Economía Institucional, 8 (14). Recuperado de: http://redalyc.uaemex.mx/pdf/419/41901406.pdf

CONAPO (2010). Estimaciones de CONAPO con base en el INEGI, Censo de Población y Vivienda 2010, Principales resultados por localidad.

Diez J. (mayo, 2004). Transformaciones en la gestión municipal: el caso latinoamericano. Ciencia para el desarrollo, grupo economía y región, (6). Recuperado de: http://www.economiayregion.com.ar/pdf/documento\%20 de\%20trabajo-JDIEZ.pdf

IDH (2009). Informe sobre Desarrollo Humano Jalisco, que refiere a estadísticas del periodo 2000-2005.

INEGI (2012). Perspectiva estadística Jalisco. Marzo 2012.

INEGI (2010). Principales resultados del Censo de Población y Vivienda 2010. Jalisco

MARTínEZ, J. (2007). Las instituciones y el desarrollo económico un análisis breve. Observatorio de la Economía Latinoamericana, (Vol 73). Recuperado de: http://www.eumed.net/cursecon/ecolat/mx/

NORTH, D.C. (1990). Institutions, Institutional Change and Economic Performance, Cambridge University Press, Cambridge.

NORTH, D. C. y THOMAS, R. (1973). The Rise of the Western World. A New Economic History. Cambridge University Press.

OLson, M. (1965). The Logic of Collective Action, Cambridge, Harvard University Press.

PED (2030). Plan Regional de Desarrollo del Estado de Jalisco, Región Norte, 2030.

PENG, M. (2010). Estrategia Global. Ed. CENGAGE Learning. Apalancando los recursos y las capacidades (pp. 71).

RODRícuez, P. (1998). Dynamics of regional growth in Europe. Clarendon Press, Oxford.

ROMERO, L. (julio 2009). Dan rumbo al norte de Jalisco. La Gaceta. UDG. Recuperado de: http://www.gaceta. udg.mx/Hemeroteca/paginas/577/G577_COT\%209.pdf

SEIJAL (2012). Sistema Estatal de Información Jalisco.

SPILleR, A. (marzo, 2010). "El Norte de Jalisco, de los más pobres". La Gaceta. UDG. Recuperado de: http:/l www.gaceta.udg.mx/Hemeroteca/paginas/605/G605_COT\%204.pdf

VÁzQUeZ BARQUERO, A. (2000), Desarrollo económico local y descentralización: Aproximación a un marco conceptual, Santiago de Chile, Comisión Económica para América Latina y el Caribe (CEPAL). 
